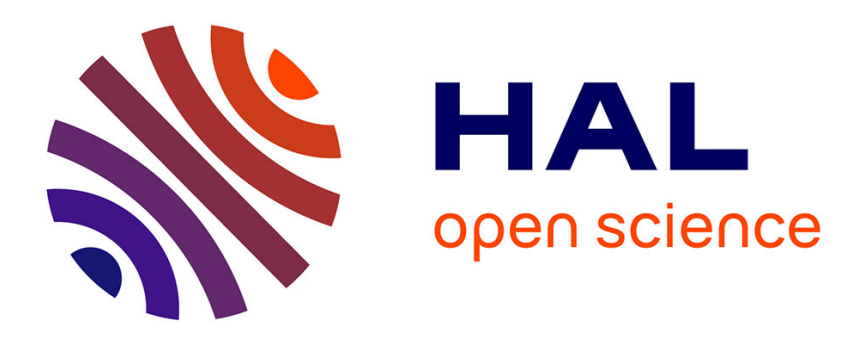

\title{
On the existence of Pairwise stable weighted networks
} Philippe Bich, Lisa Morhaim

\section{To cite this version:}

Philippe Bich, Lisa Morhaim. On the existence of Pairwise stable weighted networks. 2017. halshs01564591

\section{HAL Id: halshs-01564591 https://shs.hal.science/halshs-01564591}

Preprint submitted on 19 Jul 2017

HAL is a multi-disciplinary open access archive for the deposit and dissemination of scientific research documents, whether they are published or not. The documents may come from teaching and research institutions in France or abroad, or from public or private research centers.
L'archive ouverte pluridisciplinaire HAL, est destinée au dépôt et à la diffusion de documents scientifiques de niveau recherche, publiés ou non, émanant des établissements d'enseignement et de recherche français ou étrangers, des laboratoires publics ou privés. 


\title{
ON THE EXISTENCE OF PAIRWISE STABLE WEIGHTED NETWORKS
}

\author{
Philippe Bich* AND Lisa MORHAIM ${ }^{\dagger}$
}

Tuesday $18^{\text {th }}$ July, 2017

\begin{abstract}
We provide the existence of pairwise stable weighted networks under assumptions similar to Nash theorem. In particular, contrarily to the case of unweighted networks, the existence of closed improving cycles does not prevent the existence of Pairwise stable weighted networks. Then, we extend our existence result, allowing payoffs to depend on some game-theoretic strategies. Many applications are given.
\end{abstract}

Keywords: Pairwise Stable Network, Weighted Network.

JEL Classification: C72, D85.

*Paris School of Economics and University of Paris 1 Panthéon-Sorbonne.

${ }^{\dagger}$ University of Paris 2 Panthéon-Assas, CRED Paris Center for Law and Economics. 


\section{INTRODUCTION}

Game theory has become established as a major theory to describe, explain and predict social and economic interactions. One of the central tools of game theory, frequently used in economic analysis, was provided by Nash in $1950[19,20]$ with the notion of Nash equilibrium: it is a profile of strategies such that each player anticipates the strategies of the others, and no player has some incentive to change alone his strategy.

A version of Nash existence theorem states that in every non cooperative game whose payoffs are continuous and satisfies some concavity assumption, there exists a Nash equilibrium [8]. This simple and flexible existence theorem has contributed to the popularity of Nash equilibrium, since in many situations where people interact strategically, this makes available a possible prediction of a reasonable outcome.

More recently, there has been a growing interest for the role of networks in social and economic interactions. A network can be defined by a set $N$ of nodes (agents) and by some links between agents, measuring some (unidimensional) characteristic of the possible relationships.

An important contribution of the network literature has been to propose strategic models of network formation (e.g., Aumann and Myerson [1], Slikker and van den Nouweland [22] or Myerson [18]), ${ }^{1}$ explaining how and why agents establish bilateral links, based on payoff maximization. In particular, such models have helped to understand the emergence of specific forms of networks.

A key concept involved in network formation theory, introduced in their seminal paper by Jackson and Wolinsky [15], is pairwise stability ${ }^{2}$. Roughly, a network is pairwise stable if "no two agents could gain from linking and no single agent could gain by severing one of his or her link" (see [15]). Its main distinctive feature, compared with Nash equilibrium, is its "cooperative" aspect, since it also takes into account bilateral deviations (when links are created), although Nash equilibrium concept only considers unilateral

\footnotetext{
${ }^{1}$ For a recent survey on this subject, see Chapter 6 of "The Oxford Handbook on the Economics of Networks", by Ana Mauleon and Vincent Vannetelbosch[16]

${ }^{2}$ This is not the only stability concept used in network formation theory. For example, for networks whose weights of links are separable functions of the efforts of the agents implied in the link, Nash equilibrium is a good candidate: see [3] or [23].
} 
deviations. Beyond this difference, it is also a natural predictive solution concept. This raises the important question of its existence.

The few existence results in the literature consider unweighted ${ }^{3}$ networks. Jackson and Watts [13] have provided the first existence result for such networks. Their condition rests on improving paths (a path of networks for which at each step, the utility of one or two agents is improved by deleting or adding a link). They prove that for every payoff functions profile, either there exists a pairwise stable network, or there exists a closed improving cycle (see [14]). As a byproduct, under the assumption that there exists some network potential function, closed cycles are ruled out, and the existence of pairwise equilibria is obtained (Jackson and Watts [13]). This general approach have been extended or refined (see Chakrabarti and Gilles [7] or Hellmann [11]).

There are several reasons to be interested in weighted networks, i.e. networks for which the relationships are measured by reals. First, in many situations, this is natural to quantify the weight of a relationship in a continuous way (it can measure a debt, the strength of friendship, some level of confidence, some capacity, a geographical distance...). A second reason is tractability of continuous models: in game theory, it is well known that passing to continuous strategy spaces (through mixed strategies for example) allows to get the existence of a Nash equilibrium almost for free, which is a condition for many further developments. We will see that this is similar in network theory: passing from unweighted networks to weighted ones allows to get the existence of a pairwise stable network under weak assumptions. This should permit many developments (e.g., structure of the set of pairwise stable networks, refinement notions, conditions for uniqueness, ...).

Aim and main results of our paper

The main motivation of this paper is to prove the existence of a pairwise stable weighted network under conditions similar to Nash theorem existence result: some geometric condition (quasiconcavity of payoffs with respect to each link), and some topological condition (continuity). In particular, contrarily to the case of unweighted networks, the existence of closed improving cycles does not prevent the existence of pairwise stable weighted networks. This could be rather intuitive, since weighting the network entails some con-

\footnotetext{
${ }^{3} \mathrm{~A}$ network is unweighted if a link can be in two possible states (connection or no connection). In particular, the space of links is discrete.
} 
vexification effect. But it is unclear whether standard methods of game theory, using Best-responses, can be used to get existence. Indeed, pairwise stability concept involves both unilateral and bilateral deviations, although a standard game-theoretic approach takes into account only unilateral deviations.

More generally, we prove the existence of a Nash-pairwise stable pair $(g, y)$, which incorporates at the same time a network $g$ and some strategy profile $y=\left(y_{1}, \ldots, y_{N}\right)$, where $y_{i}$ is an individual strategy of player $i$. Roughly, a pair $(g, y)$ is Nash-pairwise stable if:

1. No player can improve strictly his payoff by changing his individual strategy $y_{i}$, the network $g$ being fixed.

2. No player can improve strictly his payoff by decreasing his link with another player, the profile of strategies being fixed.

3. Finally, no two agents could gain from increasing the weight of their common link, the profile of strategies being fixed.

The case where $y$ plays no role gives the standard pairwise stablility concept (thus in particular, our general existence result entails the existence of pairwise stable networks), and the case where $g$ plays no role gives a standard Nash existence result. Our formalism encompasses many existing models, and makes possible some endogenous explanation of network formation in most of them. For example, recent theoretical papers analyse the behaviour of contributors on public goods in some exogenous network (e.g., [4]). Our existence result opens the door to considering an additional network-formation aspect in such models.

Organization of the paper

The paper is organized as follows. After preliminaries, where pairwise stability definition is recalled (Section 2), we state our main existence result together with some examples (Section 3). An important particular case is when payoffs are affine with respect to the weight of each link, which is developed in Section 4. In Section 5, we explain why our assumptions are tight, and provide counterexamples to our existence result if some assumptions are removed. In Section 6, we provide a more general model (where links formation is explained through some efforts of players), with the existence proof. 


\section{PRELIMINARIES}

There are $N$ agents $^{4}$ which interact in a network of relationships. The strength $^{5}$ of the relationship involving two agents $i \in N$ and $j \in N$ is $g_{i j} \in[0,1]$. For example, $g_{i j}$ can measure information exchange, or time spent together. We let $\mathcal{L}:=\{(i, j) \in N \times N: i \neq j\}$ be the set of directed links, and $\mathcal{L}^{\prime}:=\{\{i, j\} \in N \times N: i \neq j\}$ be the set of undirected links. For simplicity, and when there is no risk of confusion, we will denote a link $i j$, with $i j=j i$ when $i j \in \mathcal{L}^{\prime}$ and $i j \neq j i$ when $i j \in \mathcal{L}$. Formally:

Definition 2.1. - A weighted network ${ }^{6}$ (on $N$ ) is a mapping g from $\mathcal{L}$ to $[0,1]$ such that $g(i j)=g(j i)$ for every $i j \in \mathcal{L}$. The network $g$ is unweighted if for every $i j \in \mathcal{L}, g(i j) \in\{0,1\}$.

For convenience, if $i$ and $j$ are two distinct elements of $N, g(i j)$ will be simply denoted $g_{i j}$. Throughout this paper, we let $\mathcal{G}$ be the set of weighted networks. For every $i j \in \mathcal{L}, \delta^{i j}$ is the network with only one full link between $i$ and $j$, that is $\delta_{k l}^{i j}$ is equal to 0 if $k l \neq i j$ and equal to 1 otherwise. The following definition is the natural adaptation of pairwise stability concept (Jackson and Wolinsky [15]) to weighted networks. Hereafter, we let $v_{i}: \mathcal{G} \rightarrow \mathbf{R}$ be the payoff function of each agent $i \in N$, and $v=\left(v_{i}\right)_{i \in N}$ be the profile of payoff functions. Moreover, if $g=\left(g_{i j}\right)_{i j \in \mathcal{L}}$ is a weighted network, then for every $i j \in \mathcal{L}$ and every $x \in[0,1], g^{\prime}=\left(x, g_{-i j}\right)$ denotes the weighted network such that $g_{k l}^{\prime}=g_{k l}$ for every $k l \neq i j$ and $k l \neq j i$, and $g_{i j}^{\prime}=g_{j i}^{\prime}=x$.

Definition 2.2. - A weighted network $g \in \mathcal{G}$ is said to be pairwise stable (resp. weakly pairwise stable) with respect to $v$ if:

1. for every $i j \in \mathcal{L}$, for every $x \in\left[0, g_{i j}\left[, v_{i}\left(\left(x, g_{-i j}\right)\right) \leq v_{i}(g)\right.\right.$.

2. for every ij $\in \mathcal{L}$, for every $\left.x \in] g_{i j}, 1\right]$, if $v_{i}\left(\left(x, g_{-i j}\right)\right)>v_{i}(g)$ then $v_{j}\left(\left(x, g_{-i j}\right)\right)<v_{j}(g)$ (resp. $\left.v_{j}\left(\left(x, g_{-i j}\right)\right) \leq v_{j}(g)\right)$.

\footnotetext{
${ }^{4}$ For simplicity, $N$ denotes either the set of agents, or its cardinal, and we assume $N \geq 2$.

${ }^{5}$ The normalization in $[0,1]$ is without any loss of generality. See, for example, Bloch and Dutta [3].

"Note that what we call "weighted network" is usually called "undirected weighted network" in the literature. The network would be directed if we would authorize $g(i j)$ and $g(j i)$ to be different.
} 
Thus, $g$ is pairwise stable if no two agents could gain from increasing the weight of their common link (Condition 2.) and no single agent could gain by diminishing it (Condition 1.). Remark that Condition 1 . has to be true if we reverse $i j$ into $j i$, thus it holds for both players $i$ and $j$. The difference between pairwise stability and weak pairwise stability depends on what is meant by "no two agents could gain": if the mutual gain is required to be strict for the two players, we get weak pairwise stability concept, if it is required to be strict only for one agent, we get the (standard) pairwise stability concept.

Since the seminal paper of Jackson and Wolinsky [15], pairwise stability has been one of the most popular stability concept in network literature (see, for example, Jackson and Watts [13], Goyal and Joshi [10], Calvó-Armengol and Ilkiliç [6], Hellmann [11], Miyauchi [17], Bloch and Dutta [3], and for surveys Jackson [12], Mauleon and Vannetelbosch [16]). One of its remarkable feature is that it allows to endogenize the formation of networks. Yet, there is no general existence result of weighted pairwise stable networks, in the spirit of Nash equilibrium existence result (see [8], [19] or [21]). The main objective of this paper is to provide such an existence result, to extend it to a more complex strategic framework, and to illustrate the applicability of the result.

\section{Existence OF NASH-PAIRWISE STABLE PROFILES}

We now describe the model considered in this paper, which encompasses several existing models of networks and games. Each player $i \in N$ has to choose some strategy $y_{i}$ in some strategy space $Y_{i}$. We denote by $Y=\prod_{i \in N} Y_{i}$ the set of strategy profiles of all players. We assume that the payoff function of each player $i \in N$ is a function $v_{i}: \mathcal{G} \times Y \rightarrow \mathbf{R}$. We denote by $v=$ $\left(v_{1}, \ldots, v_{N}\right)$ the profile of payoff functions.

Definition 3.1.- A network-game is a pair $(Y, v)$, where $Y$ is the set of strategy profiles, and $v=\left(v_{1}, \ldots, v_{N}\right)$ the profile of payoff functions defined on $\mathcal{G} \times Y$.

Hereafter, we recall the following standard convention: if $y=\left(y_{i}\right)_{i \in N}$ is a strategy profile, then for every $i \in N, y_{-i}$ denotes the profile of all strategies 
except strategy $y_{i}$, that is $y_{-i}=\left(y_{j}\right)_{j \in N, j \neq i}$, and the usual abuse of notation $y=\left(y_{i}, y_{-i}\right)$ will be done. Our main result will require some of the following assumptions:

(A1) Compactness and Convexity Assumption. For every $i \in N, Y_{i}$ is a compact and convex subset of some finite dimensional vector space.

(A2) Continuity Assumption. For every $i \in N$, the function $v_{i}: \mathcal{G} \times Y \rightarrow \mathbf{R}$ is continuous. ${ }^{7}$

(A3) Quasiconcavity Assumption (resp. strict Quasiconcavity Assumption).

(i) For every $(g, y) \in \mathcal{G} \times Y$ and every player $i \in N, v_{i}\left(g,\left(d_{i}, y_{-i}\right)\right)$ is assumed to be quasiconcave (resp. strictly quasiconcave) with respect to $d_{i} \in Y_{i}$.

(ii) For every $(g, y) \in \mathcal{G} \times Y$ and every $i j \in \mathcal{L}, v_{i}\left(\left(x, g_{-i j}\right), y\right)$ is assumed to be quasiconcave (resp. strictly quasiconcave) with respect to $x \in[0,1]$.

Remark 3.1. Convexity, Compactness, Continuity and Quasiconcavity assumptions are standard in Game theory, in particular to get the existence of a Nash equilibrium in strategic games.

We now define the main stability notion of the paper. Hereafter, $(Y, v)$ is a network-game:

Definition 3.2.- The pair $(g, y) \in \mathcal{G} \times Y$ is Nash-pairwise stable (resp. weakly Nash-pairwise stable) with respect to $v$ if:

1. $\forall i \in N, \forall d_{i} \in Y_{i}, v_{i}\left(g,\left(d_{i}, y_{-i}\right)\right) \leq v_{i}(g, y)$,

2. $\forall i j \in \mathcal{L}, \forall x \in\left[0, g_{i j}\left[, v_{i}\left(\left(x, g_{-i j}\right), y\right) \leq v_{i}(g, y)\right.\right.$.

3. $\left.\forall i j \in \mathcal{L}, \forall x \in] g_{i j}, 1\right], v_{i}\left(\left(x, g_{-i j}\right), y\right)>v_{i}(g, y) \Rightarrow v_{j}\left(\left(x, g_{-i j}\right), y\right)<v_{j}(g, y)$ (resp. $\left.v_{j}\left(\left(x, g_{-i j}\right), y\right) \leq v_{j}(g, y)\right)$.

If $(g, y) \in \mathcal{G} \times Y$ is Nash-pairwise stable (resp. weakly Nash-pairwise stable), we say that $g$ is a pairwise stable network (resp. a weakly pairwise stable network) associated to the strategy profile $y$.

Remark 3.2. Assertion 1. says that no player can improve his payoff by modifying his strategy $y_{i}$. Assertion 2. says that no player can improve

\footnotetext{
${ }^{7}$ The set of weighted networks $\mathcal{G}$ is a convex and compact subset of the set $\mathcal{F}(\mathcal{L}, \mathbf{R})$ of functions from $\mathcal{L}$ to $\mathbf{R}$, where $\mathcal{F}(\mathcal{L}, \mathbf{R})$ is endowed with the natural multiplication and addition, and with its natural Euclidean topology. The set $\mathcal{G}$ is endowed with the induced topology.
} 
his payoff by decreasing the weight of his relationship with another player. Assertion 3. says that there is no pair of players who can both improve their payoffs by increasing the weight of their relationship.

The proof of the following theorem can be found in the appendix.

TheOREM 3.1. - For every network-game (Y,v) satisfying (A1), (A2) and Quasiconcavity Assumption (A3) (resp. strict Quasiconcavity Assumption (A3)), there exists $(g, y) \in \mathcal{G} \times Y$ which is weakly Nash-pairwise stable (resp. Nash-pairwise stable) with respect to $v$.

If the payoff function $v_{i}$ of each player $i \in N$ goes from $\mathcal{G}$ to $\mathbf{R}$ (i.e., there is no more strategy spaces $Y_{i}$ ), then we get, as a corollary of Theorem 3.1:

THEOREM 3.2.- Let $v=\left(v_{n}\right)_{n \in N}$ be a profile of continuous payoff functions from $\mathcal{G}$ to $\mathbf{R}$ satisfying Quasiconcavity Assumption (resp. strict Quasiconcavity Assumption), then there exists some network $g \in \mathcal{G}$ which is weakly pairwise stable (resp. pairwise stable) with respect to $v$.

Proof. Define $Y_{i}=\{0\}$ for every $i \in N$, and $\tilde{v}_{i}(g, 0)=v_{i}(g)$. If $v$ is continuous and satisfies Quasiconcavity Assumption, then we can apply Theorem 3.1 to $(\tilde{v}, Y)$, which gives the existence of a weakly Nash-pairwise stable pair $(g,\{0\})$. Then $g$ is weakly pairwise stable with respect to $v$.

EXAMPle 3.1.- (A Public good Provision model)

We consider the following model introduced by Bramoullé and Kranton [4], but we add some network-formation aspect. There are $N$ agents. Agent $i$ exerts some effort $e_{i} \in\left[0, \overline{e_{i}}\right]$, where $\overline{e_{i}}>0$. Given the (endogenous) network $g \in \mathcal{G}$, the payoff function of agent $i$ is

$$
v_{i}(g, e)=b\left(e_{i}+\sum_{j \neq i} g_{i j} e_{j}\right)-c_{i} e_{i}-d_{i} \sum_{j \neq i} g_{i j}
$$

where $e=\left(e_{1}, \ldots, e_{n}\right)$ is the profile of efforts, $c_{i}>0$ is the marginal cost of effort for agent $i, d_{i}>0$ is the marginal cost of forming a link for $i$, and $b:[0,+\infty[\rightarrow[0,+\infty[$ is strictly concave and strictly increasing. When $\left(d_{1}, \ldots, d_{n}\right)=0$ and $g$ is exogenous, this is exactly the model of Bramoullé and Kranton [4].

We can apply Theorem 3.1, since the payoff functions are continuous, concave with respect to each link and concave with respect to each strategy $e_{i}$. That is, 
there exists a weakly Nash-pairwise stable pair $\left(e=\left(e_{1}, \ldots, e_{n}\right), g\right) \in\left[0, \overline{e_{i}}\right]^{N} \times$ $\mathcal{G}$. Remark that we can get the existence of a Nash-pairwise stable pair if we restrict the players to choose efforts in $\left[\underline{e_{i}}, \overline{e_{i}}\right]$, where $\underline{e_{i}}>0$ and $\overline{e_{i}}>\underline{e_{i}}$ : indeed, in this case, the payoffs are strictly-concave with respect to each link, and the strict version of Theorem 3.1 can be applied.

This extend the analysis of Bramoullé and Kranton [4], since beyond the existence of a Nash equilibrium of a profile of efforts, we get the existence of an endogenous network, associated to e, which satisfies pairwise stability condition.

EXAMPLE 3.2.- (Patent race: Goyal and Joshi [10])

We now apply our main existence result to the following extension of Goyal and Joshi Patent race model [10] to weighted networks. Consider $n$ firms who are competing for obtaining a patent. The firm which wins the race gets the patent of value 1 , the others get 0 . We let $g_{i j} \in[0,1]$ measures some possible cooperation in $R \& D$ between firm $i$ and firm $j$. Let $\tau\left(n_{i}(g)\right)$ denotes the (random) time at which firm $i$ is ready to deposit some patent: it is assumed to follow some exponential distribution whose parameter is $n_{i}(g)=$ $\sum_{j \in N-\{i\}} g_{i j}$. Thus, $P\left(\tau\left(n_{i}(g(s))\right) \leq t\right)=1-e^{-t n_{i}(g)}$, that is firm $i$ can get the patent sooner if the relationships with its neighbors increase. Assuming that the distribution of the times of innovation are independent across the firms, we get that the expected payoff of firm is (see [10]):

$$
\pi_{i}(g)=\frac{n_{i}(g)}{\rho+2 n_{i}(g)+\sum_{j \neq i} n_{j}\left(g_{-i}\right)}=\frac{g_{i j}+a_{i}(g)}{2 g_{i j}+b_{i}(g)}
$$

where $g_{-i}$ denotes the network where all links with $i$ are $0, \rho$ is the common discount factor, $a_{i}(g)=\sum_{j^{\prime} \neq j, j^{\prime} \neq i} g_{i j^{\prime}}, b_{i}(g)=\rho+2 \sum_{j^{\prime} \neq j, j^{\prime} \neq i} g_{i j^{\prime}}+\sum_{j \neq i} n_{j}\left(g_{-i}\right)$. Since we have $2 a_{i}(g)-b_{i}(g)<0$, an easy computation proves that $\pi_{i}(g)$ is strictly concave, thus satisfies strict Quasiconcavity Assumption, and Continuity is straightforward. Thus we can apply Theorem 3.2, and there exists some pairwise stable network. 


\section{Multiaffine Payoffs And MiXed EXTENSION}

\subsection{Multiaffine Payoffs}

In this section, we examine the particular case where each $v_{i}: \mathcal{G} \rightarrow \mathbf{R}$ is multiaffine, ${ }^{8}$ that is for every $i j \in \mathcal{L}$ and every $g \in \mathcal{G}, v_{i}\left(d_{i j}, g_{-i j}\right)$ is an affine function of $d_{i j} \in[0,1]$. In this case, from Theorem 3.2, there exists some weak pairwise network $g \in \mathcal{G}$. Consider the following explicit illustration:

EXAMPLE 4.1. - There are three players (the variables $x, y, z$ on the links indicate the weights of the links):

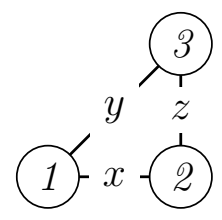

The payoffs are given by $v_{1}(x, y, z)=x\left(\frac{1}{2}-z\right)+y, v_{2}(x, y, z)=z\left(\frac{1}{2}-y\right)+x$ and $v_{3}(x, y, z)=y\left(\frac{1}{2}-x\right)+z$. Let us prove that $\left(\frac{1}{2}, \frac{1}{2}, \frac{1}{2}\right)$ is the only weak pairwise stable network. First, by Theorem 3.2, a weakly pairwise stable network $g=(x, y, z)$ exists. If $x>\frac{1}{2}$, then player 3 should decrease the weight $y$ of his link with player 1, i.e. we should have $y=0$. Then, both players 2 and 3 should increase together the weight $z$ of their common link, i.e. $z=1$. But then, player 1 should decrease the weight $x$ of his link with 2, i.e. $x=0$, which contradicts $x>\frac{1}{2}$. Since all the variables play the same roles, we finally have $x \leq \frac{1}{2}, y \leq \frac{1}{2}$ and $z \leq \frac{1}{2}$. Now, if $x<\frac{1}{2}$, then both player 1 and player 3 should increase together the weight $y$ of their common link, i.e. $y=1$, a contradiction with $y \leq \frac{1}{2}$. By symmetry, we finally have $x=y=z=\frac{1}{2}$.

EXAMPLE 4.2.- (A Two-way flow model)

We now adapt a model of Bala and Goyal [2] to weighted networks. We

\footnotetext{
${ }^{8}$ We could treat similarly the case $v_{i}: \mathcal{G} \times Y \rightarrow \mathbf{R}$, where $Y$ is the space of strategy profiles, and where $v_{i}(g, y)$ is affine with respect to each weight $g_{i j}$.
} 
define $n_{i}(g)=\sum_{j \in N-\{i\}} g_{i j}$ the sum of the weights of all direct links from agent $i$ to another agent. For every $j \neq i$, a path from $i$ to $j$ is a finite sequence $x_{0}=i, x_{1}, \ldots, x_{k}=j$ of distinct elements of $N$. Let $\mathcal{P}_{j}$ be the (finite) set of all paths from $i$ to $j$, and $n_{i}^{\prime}(g)$ be the sum on all paths in $\mathcal{P}_{j}$ and on all $j \neq i$ of the product of weights along such paths. We can interpret $n_{i}^{\prime}(g)$ as the benefit that agent $i$ receives from his links, and $n_{i}(g)$ as the cost of maintaining his links.

For every agent $i$, define

$$
v_{i}(g)=n_{i}^{\prime}(g)-c_{i} n_{i}(g)
$$

where $c_{i}>0$ is the marginal cost of maintaining the links of player $i$.

The payoffs are multiaffine, thus there exists some weak pairwise equilibrium. For example, assume there are 3 players, and consider the network $g=$ $(x, y, z)$ in Example 4.1. In this case, we find $v_{1}(x, y, z)=(x+y)\left(z+1-c_{1}\right)$, $v_{2}(x, y, z)=(x+z)\left(y+1-c_{2}\right)$ and $v_{3}(x, y, z)=(y+z)\left(x+1-c_{3}\right)$. If $c_{1}<1, c_{2}<1$ anc $c_{3}<1$, then $(1,1,1)$ is the only pairwise stable network, which corresponds to the case where everybody fully connect to each other. If $\left.c_{1} \in\right] 1,2\left[, c_{2} \in\right] 1,2\left[\right.$ and $\left.c_{3} \in\right] 1,2[$, then $(0,0,0)$ and $(1,1,1)$ are pairwise stable (no connection at all or full connection), but interestingly, $\left(x=c_{3}-\right.$ $\left.1, y=c_{2}-1, z=c_{1}-1\right)$ is another pairwise stable network which cannot exist in the unweighted model. It corresponds to the level of weights such that no player has some possibility to modify its payoff. Also, if $c_{1}>2, c_{2}>2$ and $c_{3}>2$, then $(0,0,0)$ is the only pairwise stable network. Remark that many intermediary cases are possible, which we do not explicit.

EXAMPLE 4.3.- (Information transmission)

We extend to weighted networks an information transmission model due to Calvó-Armengol [5]. Hereafter, we follow the presentation of Calvó-Armengol and Ilkiliç [6]. There are $N$ agents. If agent $i$ and agent $j$ are in a full relationship $\left(g_{i j}=1\right)$, some information can be transmitted from one player to another player, with some probability $p_{i j}$. We assume that if the relationship is weighted $\left(g_{i j} \in[0,1]\right)$, the probability of transmission is $g_{i j} p_{i j}$. Payoff of player $i \in N$ is defined by

$$
v_{i}(g)=1-\prod_{j \in N-\{i\}}\left(1-p_{j i} g_{i j}\right)-c n_{i}(g),
$$


where $g$ is a weighted network on $N, c>0, n_{i}(g)=\sum_{j \in N-\{i\}} g_{i j}$. The first term corresponds to the probability that the message is transmitted to player $i$, and the second term to the cost of maintaining his links. The payoffs are multiaffine, thus there exists some weak pairwise equilibrium.

\subsection{MiXeD EXTENSION}

Now, if $v$ is a profile of payoff functions defined on the set of unweighted networks, then we can define the mixed extension ${ }^{9}$ of $v$ as follows: for every fixed weighted network $g \in \mathcal{G}$, we interpret $g_{i j}$ as the probability that the (unweighted) link $i j$ is activated. Assuming mutual independence of the activation of the different links, $g$ defines some probability $P_{g}$ on the set of unweighted networks. Then, define $\bar{v}(g)$ the expected value of $v(\tilde{g}), \tilde{g}$ being some random network distributed according to $P_{g}$. More explicitely,

$$
\bar{v}_{i}(g)=\sum_{g^{\prime} \in \mathcal{G}^{\prime}} v_{i}\left(g^{\prime}\right) \Pi_{i j \in g^{\prime}} g_{i j}
$$

where $\mathcal{G}^{\prime}$ is the set of unweighted networks.

Theorem 3.1 implies the existence of some weak pairwise stable network. In addition, if $c_{i}: \mathcal{G} \rightarrow \mathbf{R}$ is a function associating to each weighted network the cost of formation of this network for player $i$, and if $c_{i}$ is assumed to be convex (resp. strictly convex) with respect to each variable $g_{i j}$, and continuous with respect to $g$, then we also get the existence of some weak pairwise stable network (resp. pairwise stable network) with respect to the payoffs $w_{i}(g)=\bar{v}_{i}(g)-c_{i}(g)$.

EXAMPLE 4.4. - As an illustration with two players, consider the unweighted network such that $v_{i}(g)=0$ if $g$ is the empty network, and $v_{i}(g)=1$ if the players are linked together. For every $x \in[0,1]$, define $c_{i}(x)=\frac{\alpha_{i}}{2} x^{2}, \alpha_{i}>1$. Then $w_{i}(x)=\bar{v}_{i}(x)-c_{i}(x)=x-\frac{\alpha_{i}}{2} x^{2}$, and the (unique) pairwise stable network is $\min \left\{\frac{1}{\alpha_{1}}, \frac{1}{\alpha_{2}}\right\}$. Indeed, if $x$ is a pairwise stable network, and if $i$ is a player such that $\frac{1}{\alpha_{i}}=\min \left\{\frac{1}{\alpha_{1}}, \frac{1}{\alpha_{2}}\right\}$, then either $x>\frac{1}{\alpha_{i}}$ and player $i$ should decrease strictly the weight $x$, or $x<\frac{1}{\alpha_{i}}$, and then both players should strictly

\footnotetext{
${ }^{9}$ This terminology is chosen by analogy with the mixed extension of a finite game.
} 
increase the weight $x$ to $x=\frac{1}{\alpha_{i}}$. Thus, the pairwise stable network is dictated by the player for which the cost of formation of the link is the highest.

\section{EXAMPLE 4.5.- (Coordination Games)}

In [9] (cf p.66), Goyal considers some coordination game on a network, where each player plays a coordination game with each of her neighbors. We extend his model to capture also the formation of the network. First, consider a twoaction coordination game among two players, summarized by the following $2 \times 2$ matrix:

\begin{tabular}{|c|c|c|}
\hline & $\alpha$ & $\beta$ \\
\hline$\alpha$ & $(a, a)$ & $(d, d)$ \\
\hline$\beta$ & $(e, d)$ & $(b, b)$ \\
\hline
\end{tabular}

Parameters are assumed to satisfy $a>d, b>d, d>e$ and $a+d>b+e$, so that there are gains from choosing the same action, but rewards may differ depending on which actions the players coordinate on. Let $\pi_{i}(x, y)$ be the payoff of player $i$ when he chooses action $x$ while her opponent chooses action $y$.

There are two pure strategy strict Nash equilibria $(\alpha, \alpha)$ and $(\beta, \beta)$, and coordinating on one of them is better than not coordinating at all. We define some social coordination game as follows: there are $N=\{1,2, \ldots, n\}$ players linked through an undirected weighted network $g \in \mathcal{G}$. As discussed in the mixed extension subsection, each weight $g_{i j}$ is interpreted as the probability that the (full) link ij is activated, the different probabilities being mutually independent. Each player $i$ plays the coordination game with each of her neighbors, which are randomly chosen according to the probabilities defined by $g$. Let $\sigma_{i}$ be the mixed strategy of player $i \in N, \Delta_{i}$ its mixed strategy set and $\Delta=\prod_{i \in N} \Delta_{i}$ denote the set of mixed strategy profiles of all players in the network. The expected payoff of player $i$ is $\sum_{j \in N-\{i\}} g_{i j} \pi_{i}\left(\sigma_{i}, \sigma_{j}\right)$. We can apply Theorem 3.1, since the payoff functions are continuous and satisfy Quasiconcavity Assumption (they are multiaffine). Thus, we get the existence of a weakly Nash-pairwise stable pair $(g, \sigma)$. 


\section{Some Counterexamples}

\subsection{Continuity is NeCESSARY FOR THE EXISTence of A WEAK PAIRWISE STABLE NETWORK}

Consider two players, $x \in[0,1]$ being the weight of the link between them. The payoffs are defined for $i=1,2$ by $v_{i}(x)=-x$ if $x \neq 0$ and $v_{i}(0)=-1$. Then $x>0$ is not pairwise stable, since some player (in fact both) can increase his payoff by decreasing the weight $x>0$ of the link. But $x=0$ is not pairwise stable, since both players can increase strictly their payoff by choosing $x=\frac{1}{2}$. Thus, there is no pairwise stable network, although the payoffs are quasiconcave.

\subsection{QUASICONCAVITY IS NECESSARY FOR THE EXISTENCE OF A WEAK PAIRWISE STABLE NETWORK}

The following example illustrate why quasiconcavity is, in general necessary. Again, consider two players, $x \in[0,1]$ being the weight of the link between them. Let

$$
\begin{aligned}
& v_{1}(x)= \begin{cases}\frac{1}{3}-x & \text { if } x \in\left[0, \frac{1}{3}\right], \\
x-\frac{1}{3} & \text { if } x \in\left[\frac{1}{3}, 1\right]\end{cases} \\
& v_{2}(x)= \begin{cases}\frac{1}{3}+2 x & \text { if } x \in\left[0, \frac{1}{3}\right] \\
\frac{7}{6}-\frac{x}{2} & \text { if } x \in\left[\frac{1}{3}, 1\right]\end{cases}
\end{aligned}
$$

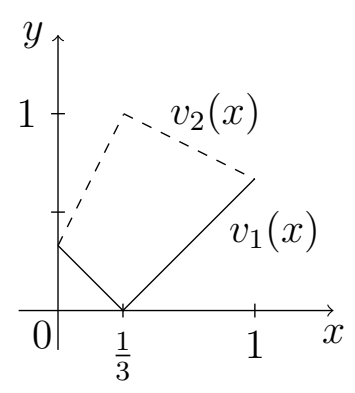

The network $x=0$ is not pairwise stable, because $x=1$ is strictly better for both player. Similarly, any $\left.x \in] 0, \frac{1}{3}\right]$ is not pairwise stable because $x=0$ is strictly better for player 1 . Last, $\left.x \in] \frac{1}{3}, 1\right]$ is not pairwise stable because $x=\frac{1}{3}$ is strictly better for player 2 . 


\subsection{StRICT QUASICONCAVITY IS NECESSARY FOR THE EX- ISTENCE OF A PAIRWISE STABLE NETWORK}

Consider Example 4.1: it has been proven that $\left(\frac{1}{2}, \frac{1}{2}, \frac{1}{2}\right)$ is the only weak pairwise stable network. It is not pairwise stable, because if player 1 and 2 both increase $x=\frac{1}{2}$, player 1's payoff is unchanged, but player 2's payoff increases strictly. In this example, Strict Quasiconcavity Assumption does not hold, for example when $x=\frac{1}{2}$.

\section{Appendix: proof of Theorem 3.1}

\subsection{A MORE GENERAL RESULT}

We shall prove a more general result (see Theorem 6.1 below), which could be interesting in itself. Then, in the next subsection, Theorem 3.1 will be proved to be an immediate consequence of Theorem 6.1.

In the spirit of Bloch and Dutta [3], for every $i j \in \mathcal{L}$, we now consider $X_{i j}$, some set of amounts of resources (e.g. time or money) invested by player $i$ in his relationship with $j$. Thus, each player $i \in N$ chooses some vector $x_{i}=\left(x_{i j}\right)_{j \in N-\{i\}}$, specifying the amount of resources invested in the relationship with all the other players. We denote by $X:=\prod_{i j \in \mathcal{L}} X_{i j}$ the set of all possible resource profiles. For every $i j \in \mathcal{L}, x_{i j}$ and $x_{j i}$ induce some weight of the link $i j$, denoted $\phi_{i j}\left(x_{i j}, x_{i j}\right)$, where $\phi_{i j}: X_{i j} \times X_{j i} \rightarrow[0,1]$.

DeFINITION 6.1.- The network formation mapping is the mapping $\phi: X \rightarrow$ $\mathcal{G}$ defined by: for every $i j \in \mathcal{L}, \phi(x)_{i j}=\phi_{i j}\left(x_{i j}, x_{i j}\right)$.

Second, each player $i \in N$ has to choose some strategy $y_{i}$ in his strategy space $Y_{i}$. We denote by $Y=\prod_{i \in N} Y_{i}$ the set of strategy profiles of all players. We assume that the payoff function $u_{i}: X \times Y \rightarrow \mathbf{R}$ of each player $i \in N$ only depends on the network formed according to $x$ and on the strategy profile $y \in Y$ : formally, this means that for every $i \in N$, there exists some mapping $v_{i}: \mathcal{G} \times Y \rightarrow \mathbf{R}$ (we call here network payoff of player $i$, to distinguish it from $\left.u_{i}\right)$, such that for every $(x, y) \in X \times Y, u_{i}(x, y)=v_{i}(\phi(x), y)$. We denote by $v=\left(v_{1}, \ldots, v_{N}\right)$ the profile of network payoff functions, and $u=\left(u_{1}, \ldots, u_{N}\right)$ the profile of associated payoff functions. 
Definition 6.2.- A network-game is a 4-uple $(X, Y, v, \phi)$, where $X$ is the set of resource profiles, $Y$ the set of strategy profiles, $v=\left(v_{1}, \ldots, v_{N}\right)$ the profile of network payoff functions defined on $\mathcal{G} \times Y$, and $\phi: X \rightarrow \mathcal{G}$ the network formation mapping.

This allows to define some profile of payoff functions $u=\left(u_{1}, \ldots, u_{N}\right)$ by $u_{i}(x, y)=v_{i}(\phi(x), y)$ for every $i \in N$.

Hereafter, we extend the standard convention of game theory to profiles of efforts: if $x=\left(x_{k l}\right)_{k l \in \mathcal{L}}$ is a profil of efforts, then for every $i j \in \mathcal{L}, x_{-i j}$ denotes the profile of all efforts, where $x_{i j}$ has been removed from $x$, and we denote $x=\left(x_{i j}, x_{-i j}\right)$. Similarly, $x_{-i j-j i}$ denotes the profile of all efforts, where $x_{i j}$ and $x_{j i}$ have been removed from $x$, and we denote $x=\left(x_{i j}, x_{j i}, x_{-i j-j i}\right)$.

Our existence result will require some of the following assumptions:

(B1) Compactness and Convexity Assumption. For every $i j \in \mathcal{L}, X_{i j}$ and $Y_{i}$ are compact and convex subsets of finite dimensional vector spaces.

(B2) Continuity Assumption. The functions $v_{i}: \mathcal{G} \times Y \rightarrow \mathbf{R}, i \in N$, and $\phi$ are continuous.

(B3) Regularity Assumption. The network formation mapping $\phi$ is regular in the following sense: the multivalued mapping $g \in \mathcal{G} \rightarrow \phi^{-1}(g):=\{x \in X$ : $\phi(x)=g\}$ is lower semicontinuous. ${ }^{10}$

(B4) Quasiconcavity Assumption (resp. strict Quasiconcavity Assumption).

(i) For every $(x, y) \in X \times Y$ and every player $i \in N, u_{i}\left(x,\left(d_{i}, y_{-i}\right)\right)$ is assumed to be quasiconcave (resp. strictly quasiconcave) with respect to $d_{i} \in Y_{i}$.

(ii) For every $(x, y) \in X \times Y$ and every $i j \in \mathcal{L}^{\prime}, u_{i}\left(\left(d_{i j}, x_{-i j}\right), y\right)$ is assumed to be quasiconcave (resp. strictly quasiconcave) with respect to $d_{i j} \in X_{i j}$.

The following proposition shows that Assumption (B3) covers important examples of the literature.

Proposition 6.1.- i) Assume for every $i j \in \mathcal{L}, X_{i j}=X_{i j}=[0,1]$ and $\phi_{i j}\left(x_{i j}, x_{j i}\right)=\min \left\{x_{i j}, x_{j i}\right\}$ or $\phi_{i j}\left(x_{i j}, x_{j i}\right)=\max \left\{x_{i j}, x_{j i}\right\}$, then the network formation mapping $\phi$ satisfies Regularity Assumption (B3).

\footnotetext{
${ }^{10} \mathrm{~A}$ multivalued mapping $\phi$ from a topological space $A$ and valued in another topological vector space $B$ is said to be lower semicontinuous if for every open subset $V$ of $B$, the set $\{a \in A: \Phi(a) \cap V \neq \emptyset\}$ is an open subset of $A$.
} 
ii) Assume for every $i j \in \mathcal{L}, X_{i j}=\left[0,+\infty\left[\right.\right.$, and $\phi_{i j}\left(x_{i j}, x_{j i}\right)=f\left(x_{i j}\right)+f\left(x_{j i}\right)$ where $f:\left[0,+\infty\left[\rightarrow\left[0, \frac{1}{2}\right]\right.\right.$ is some continuous and invertible function (see, for example, [3]). Then the network formation mapping $\phi$ satisfies Regularity Assumption (B3).

Proof. Part i) Continuity is straightforward. To prove lower semicontinuity of $\phi^{-1}$, we only have to prove that $\phi_{i j}^{-1}$ is a lower semicontinuous multivalued function. We prove it in the minimum case, the maximum case being similar. Define $\Phi(\alpha)=\phi_{i j}^{-1}(\alpha)=\left\{\left(x_{i j}, x_{j i}\right) \in[0,1] \times[0,1]: \min \left\{x_{i j}, x_{j i}\right\}=\alpha\right\}$ for every $\alpha \in[0,1]$, and let $\left(x_{i j}, x_{j i}\right) \in \Phi(\alpha) \cap V$ for some $\alpha \in[0,1]$ and some open subset $V$ of $[0,1] \times[0,1]$, that is $\min \left\{x_{i j}, x_{j i}\right\}=\alpha$. If the minimum is reached at a unique point, for example because $x_{i j}<x_{j i}$, then, we can take $\alpha^{\prime}$ close enough to $\alpha$ (in $[0,1]$ ) so that $\left(\alpha^{\prime}, x_{j i}\right) \in V$ and $\min \left\{\alpha^{\prime}, x_{j i}\right\}=\alpha^{\prime}$, thus in that case $\left(\alpha^{\prime}, x_{j i}\right) \in \Phi\left(\alpha^{\prime}\right) \cap V \neq \emptyset$. If the minimum is reached at $x_{i j}=x_{j i}$, then $\left(\alpha^{\prime}, \alpha^{\prime}\right) \in \Phi\left(\alpha^{\prime}\right) \cap V \neq \emptyset$ for every $\alpha^{\prime}$ in some neighborhood of $\alpha=x_{i j}=x_{j i}$. This finishes the proof.

Proof. Part ii) Continuity is straightforward. Now, if $\Phi(\alpha)=\phi_{i j}^{-1}(\alpha)=$ $\left\{\left(x_{i j}, x_{j i}\right) \in\left[0,+\infty\left[\times\left[0,+\infty\left[: f\left(x_{i j}\right)+f\left(x_{j i}\right)=\alpha\right\}\right.\right.\right.\right.$ for every $\alpha \in \mathbf{R}$, choose $\left(x_{i j}, x_{j i}\right) \in \Phi(\alpha) \cap V$ for some $\alpha \in \mathbf{R}$ and some open subset $V$ of $[0,+\infty[\times[0,+\infty[$. Let us fix $x_{j i}$. For every $\alpha^{\prime} \in[0,+\infty$ [ in some neighborhood (in $[0,+\infty[$ ) of $\alpha$, the equation (in $x_{i j}$ )

$$
f\left(x_{i j}\right)+f\left(x_{j i}\right)=\alpha^{\prime}
$$

has a continuous solution $x_{i j}\left(\alpha^{\prime}\right)=f^{-1}\left(\alpha^{\prime}-f\left(x_{i j}\right)\right)$, where $f^{-1}$ is the inverse bijection of $f$ (and is well defined on some neighborhood of $\alpha-f\left(x_{i j}\right)$ ). Thus, for every $\alpha^{\prime}$ in some neighborhood of $\alpha,\left(x_{i j}\left(\alpha^{\prime}\right), x_{j i}\right) \in \Phi\left(\alpha^{\prime}\right) \cap V \neq \emptyset$, which proves regularity.

The following stability notion generalizes Nash-pairwise stability defined before. The main difference is that it considers pairs of ressource profiles and strategy profiles, instead of networks and strategy profile. Hereafter, $(X, Y, v, \phi)$ is a network-game, and $u$ is the profile of payoff functions associated to $v$.

Definition 6.3.- The pair $(x, y) \in X \times Y$ is Nash-pairwise stable (resp. weakly Nash-pairwise stable) with respect to $u$ if

1. $\forall i \in N, \forall d_{i} \in Y_{i}, u_{i}\left(x,\left(d_{i}, y_{-i}\right)\right) \leq u_{i}(x, y)$,

2. $\forall i j \in \mathcal{L}, \forall d_{i j} \in X_{i j}, \phi_{i j}\left(d_{i j}, x_{j i}\right)<\phi_{i j}\left(x_{i j}, x_{j i}\right) \Rightarrow u_{i}\left(\left(d_{i j}, x_{-i j}\right), y\right) \leq$ $u_{i}(x, y)$ 
3. $\forall i j \in \mathcal{L}, \forall d_{i j} \in X_{i j}$ and $d_{j i} \in X_{j i}$ if $u_{i}\left(\left(d_{i j}, d_{j i}, x_{-i j, j i}\right), y\right)>u_{i}(x, y)$ then $u_{j}\left(\left(d_{i j}, d_{j i}, x_{-i j, j i}\right), y\right)<u_{j}(x, y)\left(\operatorname{resp} . u_{j}\left(\left(d_{i j}, d_{j i}, x_{-i j, j i}\right), y\right) \leq u_{j}(x, y).\right)$

If $(x, y)$ is Nash-pairwise stable, we will say also that $\phi(x)$ is a pairwise stable network associated to strategy profile $y$.

THEOREM 6.1. - Let $(X, Y, v, \phi)$ be a network-game satisfying Continuity and Regularity Assumption, and $u$ be the profile of associated payoff functions.

1. Under Quasiconcavity assumption, there exists $(x, y) \in X \times Y$ which is weakly Nash-pairwise stable with respect to $u$.

2. Under Strict Quasiconcavity assumption, there exists $(x, y) \in X \times Y$ which is Nash-pairwise stable with respect to $u$.

\section{Proof of Theorem 6.1.}

The proof consists in 6 steps: in Step one, we prove that we can restrict to some convex and compact set $\mathcal{G}^{\prime}$ to find Nash-Pairwise stable pairs, $\mathcal{G}^{\prime}$ being a set of pairs $(g, y)$ of networks $g$ and of strategy profiles $y$. In Step 2, under Quasiconcavity assumption, we assume (by contradiction) that the set of weakly Nash-Pairwise stable pairs $(x, y)$ is empty. This permits to define a multivalued function $\Phi$ from $\mathcal{G}^{\prime}$ to $\mathcal{G}^{\prime}$ which associates to every element $(g, y) \in \mathcal{G}^{\prime}$ the set of $\left(g^{\prime}, y^{\prime}\right) \in \mathcal{G}^{\prime}$ resulting from "profitable deviations" from $(g, y)$. Then, we prove that $\Phi$ is a lower semicontinuous multivalued function (Step 3), and satisfies some convexity property (Step 4). Applying some general existence theorem (Step 5), we get the existence of a maximal element $(g, y) \in \mathcal{G}^{\prime}$ of $\Phi$, that is $\Phi(g, y)=\emptyset$. But to such maximal element should be associated a weakly Nash-pairwise stable pair $(x, y)$ (by definition), which provides a contradiction with the initial assumption in Step 2, and finally proves the existence of a weakly Nash-pairwise stable pair. To finish, in Step 6, we prove that under strict quasiconcavity assumption, each weakly Nash-pairwise stable pair is Nash-pairwise stable. We now begin the proof.

Step 1. Recall that each component $\phi_{i j}$ of $\phi$ is a continuous function from some compact and convex set $X_{i j} \times X_{j i}$ into $[0,1]$. Thus, for every $i j \in \mathcal{L}^{\prime}$ there exists some closed interval $\left[a_{i j}, b_{i j}\right] \subset[0,1]$ such that $\phi$ maps $X$ onto $\prod_{i j \in \mathcal{L}^{\prime}}\left[a_{i j}, b_{i j}\right]$. Now, define $\mathcal{G}^{\prime}:=\prod_{i j \in \mathcal{L}^{\prime}}\left[a_{i j}, b_{i j}\right] \times \prod_{i \in N} Y_{i}$. For every $(g, y) \in$ $\mathcal{G}^{\prime}, g$ defines an undirected weighted network and $y$ a profile of strategies.

We first assume Continuity, Regularity and Quasiconcavity assumption. To prove the existence of a weakly Nash-pairwise stable pair $(x, y) \in X \times Y$, we 
now assume, by contradiction, that there is no such weakly Nash-pairwise stable pair.

Step 2. Consider the multivalued function $\Phi: \mathcal{G}^{\prime} \rightarrow \mathcal{G}^{\prime}$ defined as follows: for every $(g, y) \in \mathcal{G}^{\prime},\left(g^{\prime}, y^{\prime}\right) \in \Phi(g, y)$ if and only if there exists some profile of resources $x=\left(x_{i j}\right)_{i j \in \mathcal{L}}$ with $g=\phi(x)$ such that one has:

(a) either $g^{\prime}=g$ and there exists some $i \in N$ and some $d_{i} \in Y_{i}$ such that $y^{\prime}=\left(d_{i}, y_{-i}\right)$ and $u_{i}\left(x,\left(d_{i}, y_{-i}\right)\right)>u_{i}(x, y)$.

(b) or $y^{\prime}=y$ and there exists some $i j \in \mathcal{L}, d_{i j} \in X_{i j}$ and $d_{j i} \in X_{j i}$ such that

(i) or (ii) below are true:

(i) Either $\phi_{i j}\left(d_{i j}, x_{j i}\right)<\phi_{i j}\left(x_{i j}, x_{j i}\right), g^{\prime}=\phi\left(d_{i j}, x_{-i j}\right)$ and $u_{i}\left(\left(d_{i j}, x_{-i j}\right), y\right)>$ $u_{i}(x, y)$

(ii) or $g^{\prime}=\phi\left(d_{i j}, d_{j i}, x_{-i j-j i}\right), u_{i}\left(\left(d_{i j}, d_{j i}, x_{-i j-j i}\right), y\right)>u_{i}(x, y)$ and $u_{j}\left(\left(d_{i j}, d_{j i}, x_{-i j-j i}\right), y\right)>u_{j}(x, y)$.

Step 3. $\Phi$ is a lower semicontinuous multivalued function, that is, for every open subset $V$ of $\mathcal{G}^{\prime}$, the set $\left\{(g, y) \in \mathcal{G}^{\prime}: \Phi(g, y) \cap V \neq \emptyset\right\}$ is an open subset of $\mathcal{G}^{\prime}$. To prove it, recall that lower semicontinuity at $(g, y) \in \mathcal{G}^{\prime}$ can be characterized as follows: for every sequence $\left(g^{n}, y^{n}\right)$ in $\mathcal{G}^{\prime}$ converging to $(g, y) \in \mathcal{G}^{\prime}$ and every $\left(g^{\prime}, y^{\prime}\right) \in \Phi(g, y)$, there exists a subsequence $\left(g^{\psi(n)}, y^{\psi(n)}\right)$ of $\left(g^{n}, y^{n}\right)$ and $\left(g^{\prime n}, y^{\prime n}\right) \in \Phi\left(g^{\psi(n)}, y^{\psi(n)}\right)$ such that $\left(g^{\prime n}, y^{\prime n}\right)$ converges to $\left(g^{\prime}, y^{\prime}\right)$.

To prove this is satisfied by $\Phi$, consider a sequence $\left(g^{n}, y^{n}\right)$ in $\mathcal{G}^{\prime}$ converging to $(g, y) \in \mathcal{G}^{\prime}$ and let $\left(g^{\prime}, y^{\prime}\right) \in \Phi(g, y)$. By definition of $\mathcal{G}^{\prime}$, there exists some $x \in X$ such that $g=\phi(x)$. We consider the following different cases:

Case 1. Assume we are in the first case (a) above, that is $g^{\prime}=g$ and $y^{\prime}=\left(d_{i}, y_{-i}\right)$ for some $i \in N$ and some $d_{i} \in Y_{i}$ such that $u_{i}\left(x,\left(d_{i}, y_{-i}\right)\right)>$ $u_{i}(x, y)$. From regularity assumption satisfied by $\phi$, and since $x \in \phi^{-1}(g)$, there exists a sequence $\left(x^{n}\right)$ converging to $x$ and a subsequence $\left(g^{\psi(n)}\right)$ of $\left(g^{n}\right)$ such that $g^{\psi(n)}=\phi\left(x^{n}\right)$ for every $n$. Define $g^{\prime n}=g^{\psi(n)}$ and $y^{\prime n}=$ $\left(d_{i}, y_{-i}^{n}\right)$. Then $\left(g^{\prime n}, y^{\prime n}\right)$ in $\mathcal{G}^{\prime}$ converges to $\left(g^{\prime}, y^{\prime}\right)$, and by continuity of $u_{i}$, for $n$ large enough, $u_{i}\left(x^{n},\left(d_{i}, y_{-i}^{\psi(n)}\right)\right)>u_{i}\left(x^{n}, y^{\psi(n)}\right)$, thus $\left(g^{\prime n}, y^{\prime n}\right)=$ $\left(\phi\left(x^{n}\right),\left(d_{i}, y_{-i}^{\psi(n)}\right)\right) \in \Phi\left(g^{\psi(n)}, y^{\psi(n)}\right)$.

Case 2. Assume we are in the second case (b) above, that is $y^{\prime}=y$ and there exists some $i j \in \mathcal{L}$ and $d_{i j} \in X_{i j}$ such that (i) or (ii) above are true.

Subcase 1. First assume (i) is true, that is $\phi_{i j}\left(d_{i j}, x_{j i}\right)<\phi_{i j}\left(x_{i j}, x_{j i}\right), g^{\prime}=$ 
$\phi\left(d_{i j}, x_{-i j}\right)$ and $u_{i}\left(\left(d_{i j}, x_{-i j}\right), y\right)>u_{i}(x, y)$

From regularity assumption satisfied by $\phi$, and since $x \in \phi^{-1}(g)$, there exists a sequence $\left(x^{n}\right)$ converging to $x$ and a subsequence $\left(g^{\psi(n)}\right)$ of $\left(g^{n}\right)$ such that $g^{\psi(n)}=\phi\left(x^{n}\right)$ for every $n$. Then define $g^{\prime n}=\phi\left(d^{\psi(n)}\right)$ where $d^{n}=\left(d_{i j}, x_{-i j}^{n}\right)$. Last, define $y^{\prime n}=y^{\psi(n)}$. By continuity, $g^{\prime n}$ converges to $g^{\prime}$ and we will have $\left(g^{\prime n}, y^{\prime n}\right) \in \Phi\left(g^{\psi(n)}, y^{\psi(n)}\right)$ for $n$ large enough, where $\left(g^{\prime n}, y^{\prime n}\right)$ satisfies Case (b) subcase (i) in the definition of $\Phi$. This ends the proof of Subcase 1, and the proof of Subcase 2 is similar.

Step 4. Let us show that for every $(g, y) \in \mathcal{G}^{\prime},(g, y) \notin \operatorname{co} \Phi(g, y)$, where co stands for the standard convex hull. By contradiction, assume that there exists $(g, y) \in \mathcal{G}^{\prime}$ such that

$$
(g, y)=\sum_{l \in L} \lambda_{l}\left(g^{l}, y^{l}\right)
$$

with $\left(g^{l}, y^{l}\right) \in \Phi(g, y), \lambda_{l} \geq 0$ for every $l \in L$ (some finite set of indexes) and $\sum_{l \in L} \lambda_{l}=1$. For $l \in L$ given, $\left(g^{l}, y^{l}\right) \in \Phi(g, y)$ means that $\left(g^{l}, y^{l}\right)$ satisfies Condition (a) above or Condition (b) above. Let $L^{\prime}$ be the set of indexes such that $\left(g^{l}, x^{l}\right)$ satisfies condition (a), and $L^{\prime \prime}$ the set of indexes such that $\left(g^{l}, y^{l}\right)$ satisfies condition (b) (remark that both conditions cannot be satisfied by a same $\left(g^{l}, y^{l}\right)$ at the same time). First, assume that there exists some $l \in L^{\prime}$ such that $\lambda_{l} \neq 0$. By definition of $L^{\prime}$ and $L^{\prime \prime}$, we have $g^{l}=g$ and $y^{l} \neq y$ for every $l \in L^{\prime}$, and $y^{l}=y$ for every $l \in L^{\prime \prime}$. Together with Equation (6.1), that gives

$$
y=\sum_{l \in L^{\prime}} \lambda_{l} y^{l}+\sum_{l \in L^{\prime \prime}} \lambda_{l} y^{l},
$$

we get

$$
y\left(1-\sum_{l \in L^{\prime \prime}} \lambda_{l}\right)=\sum_{l \in L^{\prime}} \lambda_{l} y^{l}
$$

Thus

$$
y=\frac{\sum_{l \in L^{\prime}} \lambda_{l} y^{l}}{\left(\sum_{l \in L^{\prime}} \lambda_{l}\right)}
$$


that is $y$ is a convex combination of the $y_{l}$ for $l \in L^{\prime}$, formally $y=\sum_{l \in L^{\prime}} \mu_{l} y^{l}$ with $\mu_{l}=\frac{\lambda_{l}}{\sum_{l \in L^{\prime}} \lambda_{l}}$.

Fix some $i \in N$ such that $y_{i}^{l} \neq y_{i}$ for at least one $l \in L^{\prime}$, and let $L^{\prime}=L_{1}^{\prime} \cup L_{2}^{\prime}$, where $L_{1}^{\prime}$ is the nonempty set of $l \in L^{\prime}$ such that $y_{i}^{l} \neq y_{i}$ and $y_{-i}^{l}=y_{-i}$ and $L_{2}^{\prime}=L^{\prime}-L_{1}^{\prime}$ (thus, by definition, for every $l \in L_{2}^{\prime}, y_{i}^{l}=y_{i}$.)

From $y=\sum_{l \in L^{\prime}} \mu_{l} y^{l}$ we get

$$
y_{i}=\sum_{l \in L^{\prime}} \mu_{l} y_{i}^{l}=\sum_{l \in L_{1}^{\prime}} \mu_{l} y_{i}^{l}+\sum_{l \in L_{2}^{\prime}} \mu_{l} y_{i}^{l}
$$

that is

$$
y_{i}=\sum_{l \in L_{1}^{\prime}} \mu_{l} y_{i}^{l}+\sum_{l \in L_{2}^{\prime}} \mu_{l} y_{i}
$$

thus, as above, we get that $y_{i}$ is a convex combination of the $y_{i}^{l}, l \in L_{1}^{\prime}$. By definition of $L_{1}^{\prime}$, we have $u_{i}\left(x,\left(y_{i}^{l}, y_{-i}\right)\right)>u_{i}(x, y)$ for every $l \in L_{1}^{\prime}$, and by quasiconcavity of $u_{i}$ with respect to the strategy of player $i$, we get $u_{i}\left(x,\left(y_{i}, y_{-i}\right)\right)>u_{i}(x, y)$, a contradiction.

Thus, we can now assume that either $L^{\prime}$ is empty, or for every $l \in L^{\prime}, \lambda_{l}=0$. In particular, from Equation (6.1), we get

$$
g=\sum_{l \in L^{\prime \prime}} \lambda_{l} g^{l}
$$

which is still a convex combination, and where all $\left(g^{l}, y^{l}\right) \in \Phi(g, y)$ satisfy condition (b) above, that is for every $l \in L^{\prime \prime}, y^{l}=y$ and there exists some $i j \in \mathcal{L}$ (depending on $l$ ), $d_{i j}^{l} \in X_{i j}, d_{j i}^{l} \in X_{j i}, x^{l} \in X$ with $g=\phi\left(x^{l}\right), g_{k l}=g_{k l}^{l}$ for every $k l \neq i j$, and such that at least one of the three following properties (i), (ii), (iii) below is true:

(i) $g_{i j}^{l}=\phi_{i j}\left(d_{i j}^{l}, x_{j i}^{l}\right)<\phi_{i j}\left(x_{i j}^{l}, x_{j i}^{l}\right)=g_{i j}$ and $u_{i}\left(\left(d_{i j}^{l}, x_{-i j}^{l}\right), y\right)>u_{i}\left(x^{l}, y\right)$,

(ii) $g_{i j}^{l}=\phi_{j i}\left(d_{j i}^{l}, x_{i j}^{l}\right)<\phi_{j i}\left(x_{j i}^{l}, x_{i j}^{l}\right)=g_{i j}$ and $u_{j}\left(\left(d_{j i}^{l}, x_{-j i}^{l}\right), y\right)>u_{j}\left(x^{l}, y\right)$,

(iii) $u_{i}\left(\left(d_{i j}^{l}, d_{j i}^{l}, x_{-i j, j i}^{l}\right), y\right)>u_{i}\left(x^{l}, y\right)$ and $u_{j}\left(\left(d_{i j}^{l}, d_{j i}^{l}, x_{-i j, j i}^{l}\right), y\right)>u_{j}\left(x^{l}, y\right)$.

By definition, there exists some link $i j$ in $\mathcal{L}$ (now fixed) such that $g_{i j}^{l} \neq g_{i j}$ for some $l \in L^{\prime \prime}$. Now, consider $L_{i j}$ the set of indexes $l \in L^{\prime \prime}$ such that $g_{i j}^{l} \neq g_{i j}$. It is nonempty from our choice of $i j$. 
Since $g=\sum_{l \in L^{\prime \prime}} \lambda_{l} g^{l}$, we have $g_{i j}=\sum_{l \in L^{\prime \prime}} \lambda_{l} g_{i j}^{l}=\sum_{l \in L_{i j}} \lambda_{l} g_{i j}^{l}+\sum_{l \notin L_{i j}} \lambda_{l} g_{i j}$, thus $g_{i j}$ is a convex combination of the $g_{i j}^{l}, l \in L_{i j}$. In particular, all $g_{i j}^{l}$, $l \in L_{i j}$ cannot satisfy only Conditions (i) or Conditions (ii) above, because otherwise, $g_{i j}$ would be a convex combination of reals always strictly below $g_{i j}$.

Thus, there exists some $g_{i j}^{l}, l \in L_{i j}$, satisfying Condition (iii) above, and at least one of them (which we now fix) should satisfy $g_{i j}^{l}>g_{i j}$ (again, because $g_{i j}$ is a convex combination of the $g_{i j}^{m}, m \in L_{i j}$, and because $g_{i j}^{m}<g_{i j}$ for every $g_{i j}^{m}$ satisfying Conditions (i) or (ii).) Writing Condition (iii) above with $v_{i}$, we get

$$
v_{i}\left(g-\left(g_{i j}-g_{i j}^{l}\right) \delta^{i j}, y\right)>v_{i}(g, y) \text { and } g_{i j}^{l}>g_{i j}
$$

If all $g_{i j}^{l}, l \in L_{i j}$ satisfy Condition (iii), then since $g_{i j}$ is a convex combination of the $g_{i j}^{l}, l \in L_{i j}$, from quasiconcavity of $v_{i}$ (we could have taken $v_{j}$ ) we get $v_{i}(g, y)>v_{i}(g, y)$, a contradiction. Thus, there exists $g_{i j}^{l^{\prime}}, l^{\prime} \in L_{i j}$, satisfying let say Condition (i) above (the case where it satisfies Condition (ii) would be similar). This means

$$
v_{i}\left(g-\left(g_{i j}-g_{i j}^{l^{\prime}}\right) \delta^{i j}, y\right)>v_{i}(g, y) \text { and } g_{i j}^{l^{\prime}}<g_{i j}
$$

From Equations (6.2) and (6.3), we get that $g_{i j}$ is in the convex envelop of $g_{i j}^{l}$ and $g_{i j}^{l^{\prime}}$, and from Quasiconcavity assumption, that

$$
v_{i}(g, y)>v_{i}(g, y)
$$

which yields the contradiction. This finally proves Step 4 by contradiction.

Step 5. We now prove the existence of a weakly Nash-pairwise stable element. Apply an extension of Sonnenschein's Theorem (see [24], Theorem 4) by Yannelis and Prabhakar (see [25], Theorem 5.2), there exists $(g, y) \in \mathcal{G}^{\prime}$ such that $\Phi(g, y)=\emptyset$. By definition of $\mathcal{G}^{\prime}$, there exists $x \in X$ such that $g=\phi(x)$, and $\Phi(g, y)=\emptyset$ says that $(x, y)$ is weakly Nash-pairwise stable.

Step 6. To finish the proof, we prove that the weakly Nash-pairwise stable element $(x, y)$ is also Nash-pairwise stable when Strict Quasiconcavity is assumed. By contradiction, assume that $(x, y)$ is not Nash-pairwise stable. Thus, there exists $i j \in \mathcal{L}$ and $g_{i j}^{\prime} \in[0,1]$, such that, if $g=\phi(x)$, we have

$$
v_{i}\left(g-\left(g_{i j}-g_{i j}^{\prime}\right) \delta^{i j}, y\right)>v_{i}(g, y)
$$


and

$$
v_{j}\left(g-\left(g_{i j}-g_{i j}^{\prime}\right) \delta^{i j}, y\right)=v_{j}(g, y)
$$

where $g_{i j}^{\prime}=\phi_{i j}\left(d_{i j}, d_{j i}\right)$ for some $d_{i j} \in X_{i j}$ and $d_{j i} \in X_{j i}$.

Since $\phi_{i j}$ is continuous from the convex set $X_{i j} \times X_{j i}$ to $[0,1]$, there exists $\left(d_{i j}^{\prime}, d_{j i}^{\prime}\right) \in X_{i j} \times X_{j i}$ such that $\phi_{i j}\left(d_{i j}^{\prime}, d_{j i}^{\prime}\right)=\frac{g_{i j}^{\prime}+g_{i j}}{2}$. But from strict quasiconcavity assumption, we get

$$
u_{i}\left(\left(d_{i j}^{\prime}, d_{j i}^{\prime}, x_{-i j-j i}\right), y\right)>u_{i}(x, y)
$$

and

$$
u_{j}\left(\left(d_{i j}^{\prime}, d_{j i}^{\prime}, x_{-i j-j i}\right), y\right)>u_{j}(x, y),
$$

a contradiction with the definition of $(x, y)$ being weakly Nash-pairwise stable.

Remark 6.1. Let us briefly examine the two player case, and try to explain why a natural approach in this case cannot be easily generalized to more than 3 players. For two agents, assuming that each payoff function $u_{i}:[0,1] \rightarrow \mathbf{R}$ is continuous ${ }^{11}$ and quasiconcave, the existence of pairwise stable networks can be derived as follows: from continuity, each set $\arg \max _{x \in[0,1]} u_{i}(x)$ is nonempty and closed, thus it admits some minimum element $x_{i}$. Then $\bar{x}=\min \left\{x_{1}, x_{2}\right\}$ defines a pairwise stable network: indeed, no player has some strictly profitable deviation below $\bar{x}$ by definition of the minimum above, and the two players do not have simultaneous strictly profitable deviations, because $\bar{x}$ maximizes at least the payoff of one player. Unfortunately, passing to three agents, the same idea cannot be applied, because of discontinuity issues: for example, consider $N=\{1,2,3\}$, and consider $x$ the weight of the link between 1 and $2, y$ the weight of the link between 1 and 3 and $z$ the weight of the link between 2 and 3. Payoff function of each player $i \in N$ is assumed to be a function of $(x, y, z) \in[0,1]^{3}$ satisfying continuity and quasiconcavity assumption. A natural extension of the two player approach above should drive us to define: $f_{12}(x, y, z)=\min \left\{\min \operatorname{argmax}_{y \in[0,1]} u_{2}(x, y, z), \min \operatorname{argmax}_{x \in[0,1]} u_{1}(x, y, z)\right\}$, and similarly for $f_{13}$ and $f_{23}$, by circular permutation. Then, a candidate to be a pairwise stable network would be this defined by some fixed-point of the mapping $f=\left(f_{12}, f_{13}, f_{23}\right)$. But Brouwer's theorem cannot be invoked to get existence of fixed points, because $f$ can be discontinuous: for example, for

\footnotetext{
${ }^{11}$ This could even be relaxed into upper semicontinuity.
} 
$u_{1}(x, y, z)=x\left(y-\frac{1}{2}\right)$ and $u_{2}(x, y, z)=x$, then $f_{12}$ is discontinuous at every $(x, y, z)$ such that $y=\frac{1}{2}$. A last possible idea would be to consider some players $i j$ related to the links (and not to the nodes). But then it is unclear which kind of "aggregate" payoffs we should defined so that Nash equilibria of the game provides pairwise stable network, since such aggregate payoff should incorporates both individual payoffs of agents $i$ and $j$, and translate the specific deviations rules of pairwise stability concept.

\subsection{Proof of Theorem 3.1}

The proof is a consequence of Theorem 6.1 above: simply consider $X_{i j}=$ $X_{j i}=[0,1]$ for every $i j \in \mathcal{L}$, and define $\phi_{i j}\left(x_{i j}, x_{j i}\right)=\min \left\{x_{i j}, x_{j i}\right\}$ for every $\left(x_{i j}, x_{j i}\right) \in X_{i j} \times X_{j i}$. From Theorem 6.1 applied to the payoff functions $u_{i}(x, y)=v_{i}(\phi(x), y)$, there exists some weakly Nash-pairwise stable pair $(x, y)$. Then, $(g=\phi(x), y)$ is weakly Nash-pairwise stable. Indeed, first, the optimality of each strategy $y_{i}$ for player $i$, given $y_{-i}$ and $g$, is clear, by definition. Second, if some player $i \in N$ can strictly increase his payoff $v_{i}(g, y)$ by decreasing some $g_{i j}$ to $g_{i j}^{\prime}<g_{i j}$, then Player $i$ would have a strict interest, in the network-game defined by $u$, to change his strategy $x_{i j}$ for $x_{i j}^{\prime}=g_{i j}^{\prime}$, which contradicts $(x, y)$ being weakly Nash-pairwise stable. Third, if two players $i$ and $j$ can increase strictly their payoffs $v_{i}(g, y)$ and $v_{j}(g, y)$ by increasing $g_{i j}$ to $g_{i j}^{\prime \prime}>g_{i j}$, then, these players would have a strict interest, in the network-game defined by $u$, to change their strategy $x_{i j}$ and $x_{j i}$ for, let say, $x_{i j}^{\prime \prime}=x_{j i}^{\prime \prime}=g_{i j}^{\prime \prime}$, and again, this would contradict $(x, y)$ being weakly Nash-pairwise stable. The proof is similar to get the existence of a Nashpairwise stable pair under Strict Quasiconcavity Assumption.

\section{REFERENCES}

[1] Robert Aumann and Roger Myerson. Endogenous formation of links between players and coalitions: an application of the shapley value. The Shapley Value, pages 175-191, 1988.

[2] Venkatesh Bala and Sanjeev Goyal. A noncooperative model of network formation. Econometrica, 68(5):1181-1229, 2000. 
[3] Francis Bloch and Bhaskar Dutta. Communication networks with endogenous link strength. Games and Economic Behavior, 66(1):39-56, 2009 .

[4] Yann Bramoullé and Rachel Kranton. Public goods in networks. Journal of Economic Theory, 135(1):478-494, 2007.

[5] Antoni Calvó-Armengol. Job contact networks. Journal of economic Theory, 115(1):191-206, 2004.

[6] Antoni Calvó-Armengol and Rahmi İlkılıç. Pairwise-stability and nash equilibria in network formation. International Journal of Game Theory, 38(1):51-79, 2009.

[7] Subhadip Chakrabarti and Robert P Gilles. Network potentials. Review of Economic Design, 11(1):13-52, 2007.

[8] Irving L Glicksberg. A further generalization of the kakutani fixed point theorem, with application to nash equilibrium points. Proceedings of the American Mathematical Society, 3(1):170-174, 1952.

[9] Sanjeev Goyal. Connections: An Introduction to the Economics of Networks. Princeton University Press, 2007.

[10] Sanjeev Goyal and Sumit Joshi. Unequal connections. International Journal of Game Theory, 34(3):319-349, 2006.

[11] Tim Hellmann. On the existence and uniqueness of pairwise stable networks. International Journal of Game Theory, pages 1-27, 2013.

[12] Matthew O Jackson. A survey of network formation models: stability and efficiency. Group Formation in Economics: Networks, Clubs, and Coalitions, pages 11-49, 2005.

[13] Matthew O Jackson and Alison Watts. The existence of pairwise stable networks. Seoul Journal of Economics, 14:299-321, 2001.

[14] Matthew O Jackson and Alison Watts. The evolution of social and economic networks. Journal of Economic Theory, 106(2):265-295, 2002.

[15] Matthew O Jackson and Asher Wolinsky. A strategic model of social and economic networks. Journal of economic theory, 71(1):44-74, 1996. 
[16] Ana Mauleon and Vincent Vannetelbosch. Network formation games. In Yann Bramoullé, Andrea Galeotti, Brian Rogers, and Brian W Rogers, editors, The Oxford Handbook of the Economics of Networks. Oxford University Press, 2016.

[17] Yuhei Miyauchi. Structural estimation of pairwise stable networks with nonnegative externality. Journal of Econometrics, 195(2):224-235, 2016.

[18] Roger B Myerson. Game theory: analysis of conflict. Cambridge: Harvard University Press USA, 1991.

[19] John F Nash. Equilibrium points in n-person games. Proceedings of the national academy of sciences, 36(1):48-49, 1950.

[20] John F Nash. Non-cooperative games. Annals of mathematics, 54:286295, 1951.

[21] J. B. Rosen. Existence and uniqueness of equilibrium points for concave n-person games. Econometrica, 33:520-534, 1965.

[22] Marco Slikker and Anne van den Nouweland. A one-stage model of link formation and payoff division. Games and Economic Behavior, 34(1):153-175, 2001.

[23] Chiu Ki So. Network formation with endogenous link strength and decreasing returns to investment. Games, 7(4):40, 2016.

[24] Hugo Sonnenschein. Demand theory without transitive preferences, with applications to the theory of competitive equilibrium. in J.Chipman et al., eds., Preferences, utility and demand. New York: Harcourt Brace Jovanovich, 1971.

[25] Nicholas C Yannelis and ND Prabhakar. Existence of maximal elements and equilibria in linear topological spaces. Journal of Mathematical Economics, 12(3):233-245, 1983. 